\title{
Association between D-Dimer Levels and the Prognosis of Terminal Cancer Patients in the Last Hours of Life
}

\author{
Hwan Hee Lee, M.D., In Cheol Hwang, M.D., M.P.H., Ph.D. and Jinyoung Shin, M.D., Ph.D.* \\ Department of Family Medicine, Gil Medical Center, Gachon University College of Medicine, Incheon, "Department of Family \\ Medicine, Konkuk University Medical Center, Konkuk University School of Medicine, Seoul, Korea
}

Purpose: D-dimer levels are known to be associated with poor outcomes in patients with various cancers, but their significance at the end of life remains unclear. This study investigated D-dimer levels as a prognostic indicator for terminal cancer patients in the last hours of life. Methods: The retrospective study was conducted at a palliative care unit of a tertiary cancer center, using a database to analyze the records of patients treated from January 1, 2010 to December 31, 2018. In total, 67 terminal cancer patients with available data on Ddimer levels were included. Patients' demographic data, clinical information, and laboratory values, including D-dimer levels, were collected. Survival was analyzed using the KaplanMeier method and the log-rank test. A Cox proportional-hazards model was used to identify prognostic factors of poor survival. Results: The most common site of cancer was the lung (32.8\%) and the median survival time was 5 days. Most laboratory results, particularly D-dimer levels, deviated from the normal range. Patients with high D-dimer levels had a significantly shorter survival time than those with low D-dimer levels (4 days vs. 7 days; $\mathrm{P}=0.012$ ). In the Cox regression analysis, only a high $\mathrm{D}$-dimer level was identified as a predictor of a poor prognosis (hazard ratio, 1.83; 95\% confidence interval, 1.09 3.07). Conclusion: Our results suggest that at the very end of life, D-dimer levels may serve as a prognostic factor for survival in cancer patients.

Key Words: Fibrin fibrinogen degradation products, Hospice care, Neoplasms, Prognosis, Survival
Received October 1, 2019

Revised February 1, 2020

Accepted February 4, 2020

\section{Correspondence to}

In Cheol Hwang

Department of Family Medicine, Gil Medical Center, Gachon University College of Medicine, 1198 Guwoldong, Namdong-gu, Incheon 21565 , Korea

Tel: +82-32-460-3354

Fax: +82-32-460-3354

E-mail: ichwang0211@naver.com

\section{Jinyoung Shin}

Department of Family Medicine, Konkuk University Medical Center, Konkuk University School of Medicine, 120-1 Neungdong-ro, Gwangjin-gu,

Seoul 05030, Korea

Tel: +82-2-2030-7698

Fax: +82-2-2030-7749

E-mail: jyshin@kuh.ac.kr

Dr. Hwang and Dr. Shin contributed equally as corresponding authors.

\section{INTRODUCTION}

D-dimer is a fibrin degradation product; more specifically, it is a small protein fragment formed by the breakdown of fibrin through fibrinolysis. Its name reflects the fact that it contains crosslinked D fragments of the fibrin protein (1). D-dimer testing has been used since the 1990s to diagnose thrombosis because $\mathrm{D}$-dimers are normally not present in the blood, except when blood clots are dissolved by fibrinolysis. Thrombosis is ruled out if the D-dimer level is within the reference range, whereas an elevated $\mathrm{D}$-dimer level is considered to be a predictor of deep vein thrombosis, pulmonary embolism, and disseminated intravascular coagulation (2).

Recently, D-dimer testing has been recognized as a useful tool for predicting the progression, recurrence, and prognosis of cancer (3-7). In patients with cancer, thrombin is thought to affect neovascularization and metastasis because prothrombotic mechanisms are also activated when thrombin converts fibrinogen into fibrin (8). Several studies have demonstrated the utility of $\mathrm{D}$-dimer testing in estimating the prognosis of 
colorectal cancer $(3,4)$, survival in patients with lung cancer (5), the progression of prostate cancer (6), and survival in metastatic gastric cancer patients receiving chemotherapy (7). Therefore, it seems reasonable to postulate that $\mathrm{D}$-dimer levels may also be helpful for estimating life expectancy in patients with terminal cancer.

Accurately predicting life expectancy is especially important for helping patients with terminal cancer choose to discontinue futile life-sustaining treatment and prepare for their impending death together with family members (9). Although various prognostic tools for predicting survival have been proposed, the clinical utility of those tools for patients with terminal cancer is often limited or unreliable (10). The purpose of this study was to investigate the association between $\mathrm{D}$-dimer levels and survival in Korean patients with terminal cancer facing imminent death.

\section{METHODS}

\section{Patient selection and data collection}

We collected $78 \mathrm{D}$-dimer test results from the medical records of 78 patients who had incurable terminal cancer between January 1, 2010 and December 31, 2018 in the palliative care unit of a university hospital located in Incheon. Four of the results had been obtained from the second tests in patients who underwent $\mathrm{D}$-dimer testing twice. The second tests were for follow-up purposes, not for identifying new changes in the patients' condition. Therefore, we excluded the follow-up data from the analysis. Of the remaining $74 \mathrm{D}$-dimer test results, 5 were excluded due to errors in the medical records and 2 because the patient died on the day of the blood test. Ultimately, $67 \mathrm{D}$-dimer test results were used in the analysis. Informed consent was waived because of the retrospective nature of the

Table 1. Characteristics of Participants, by D-Dimer levels.

\begin{tabular}{|c|c|c|c|c|}
\hline & \multirow{2}{*}{ Total } & \multicolumn{2}{|c|}{ D-dimer levels } & \multirow{2}{*}{ P* } \\
\hline & & $\operatorname{Low}(<1.5 \mu \mathrm{g} / \mathrm{mL})$ & High ( $\geq 1.5 \mu \mathrm{g} / \mathrm{mL})$ & \\
\hline N & 67 & 33 & 34 & \\
\hline Age (yr) & $71.8 \pm 12.5$ & $74.8 \pm 10.0$ & $68.9 \pm 14.1$ & 0.054 \\
\hline Female & $30(44.8)$ & $12(36.4)$ & $18(52.9)$ & 0.172 \\
\hline \multicolumn{5}{|l|}{ Cancer site } \\
\hline Gastrointestinal tract & $9(13.4)$ & $3(9.1)$ & $6(17.7)$ & 0.022 \\
\hline Hepatobiliary pancreatic & $14(20.9)$ & $5(15.2)$ & $9(26.5)$ & \\
\hline Lung & $22(32.8)$ & $16(48.5)$ & $6(17.7)$ & \\
\hline Urogenital tract & $13(19.4)$ & $3(9.1)$ & $10(29.4)$ & \\
\hline Others & $9(13.4)$ & $6(18.2)$ & $3(8.8)$ & \\
\hline \multicolumn{5}{|l|}{ ECOG performance status } \\
\hline$\leq 2$ & $13(20.6)$ & $6(19.4)$ & 7 (21.9) & 0.970 \\
\hline 3 & $24(38.1)$ & $12(38.7)$ & $12(37.5)$ & \\
\hline 4 & $26(41.3)$ & $13(41.9)$ & $13(40.6)$ & \\
\hline Current infection & $32(47.8)$ & $18(54.6)$ & $14(41.2)$ & \\
\hline \multicolumn{5}{|l|}{ Laboratory findings } \\
\hline White blood cell $\left(10^{3} / \mathrm{mm}^{3}\right)$ & $14.7 \pm 9.6$ & $13.8 \pm 7.6$ & $15.6 \pm 11.2$ & 0.442 \\
\hline Hemoglobin (g/dL) & $10.4 \pm 2.4$ & $10.7 \pm 2.7$ & $10.0 \pm 2.1$ & 0.218 \\
\hline Platelet $\left(10^{3} / \mathrm{mm}^{3}\right)$ & $234 \pm 199$ & $238 \pm 114$ & $230 \pm 199$ & 0.834 \\
\hline Creatinine (mg/dL) & $1.4 \pm 2.2$ & $1.5 \pm 2.8$ & $1.2 \pm 1.2$ & 0.498 \\
\hline Albumin (g/dL) & $2.9 \pm 0.6$ & $2.9 \pm 0.7$ & $2.9 \pm 0.5$ & 0.614 \\
\hline Total bilirubin (mg/dL) & $2.2 \pm 4.3$ & $1.9 \pm 4.8$ & $2.4 \pm 3.9$ & 0.627 \\
\hline PTINR & $1.5 \pm 2.1$ & $1.2 \pm 0.2$ & $1.8 \pm 2.9$ & 0.254 \\
\hline C-reactive protein (mg/dL) & $13.6 \pm 10.0$ & $12.1 \pm 11.1$ & $15.1 \pm 8.7$ & 0.218 \\
\hline
\end{tabular}

ECOG: Eastern Cooperative Oncology Group, PT INR: prothrombin time international normalized ratio, SD: standard deviation.

Data are presented as mean \pm SD or number (\%).

*From the test or Chi-square test. 
study. The study protocol was approved by the institutional review board (GCIRB2019-149).

We reviewed each patient's age, sex, primary cancer site, Eastern Cooperative Oncology Group (ECOG) performance status, presence of infection, date of the $\mathrm{D}$-dimer test, date of death or transfer, D-dimer level, and other laboratory findings, including white blood cell count, hemoglobin, platelet count, albumin, total bilirubin, creatinine, C-reactive protein, and prothrombin time international normalized ratio. Since basic blood tests are routinely performed once every week in the palliative care unit, the results of the tests performed on the day closest to the $\mathrm{D}$-dimer test were used in the analysis. The ECOG performance status is a 5-point scale used to assess patients' daily living abilities, with 0 indicating that the patient is fully active and 4 indicating that the patient cannot perform any self-care (11). The presence of an infection was determined based on the use of antibiotics at the time of the blood test.

The survival time was calculated from the date of the $\mathrm{D}^{-}$ dimer test to the date of death. The date of in-hospital death or discharge (or transfer) was the last follow-up date. The endpoint was defined as death, with survival at the time of discharge or transfer treated as censored data.

\section{Data analysis}

The characteristics of patients are presented as mean \pm standard deviation or as number (percentage). The 2 groups stratified by $\mathrm{D}$-dimer values $(<1.5 \mu \mathrm{g} / \mathrm{mL}$ or $\leq 1.5 \mu \mathrm{g} / \mathrm{mL})$ were compared using the $\mathrm{t}$-test or chi-square test. The logrank test was used to compare differences in survival time according to age, sex, ECOG performance status, infection, leukemia, anemia, thrombocytopenia, elevated serum creatinine, hypoalbuminemia, hyperbilirubinemia, prothrombin time prolongation, C-reactive protein, and D-dimer level. KaplanMeier survival curves are presented to illustrate differences in the survival time between patients with low and high D-dimer levels. Univariate Cox proportional-hazards regression analysis was conducted to identify factors associated with a poor prognosis. STATA version SE 9 (Stata Corp, College Station, TX, USA) was used for all statistical analyses, with the threshold of statistical significance set at $\mathrm{P}<0.05$.
Table 2. Survival Time (Days) according to Patients' Characteristics ( $N=67)$.

\begin{tabular}{|c|c|c|c|}
\hline & $\begin{array}{c}\text { No. of } \\
\text { patients }\end{array}$ & $\begin{array}{c}\text { Median } \\
\text { (interquartile } \\
\text { range) }\end{array}$ & P* \\
\hline Age (yr) & & & 0.120 \\
\hline$<75$ & 35 & $4(3 \sim 8)$ & \\
\hline$\geq 75$ & 32 & $6(3 \sim 10)$ & \\
\hline Sex & & & 0.179 \\
\hline Male & 37 & $7(3 \sim 11)$ & \\
\hline Female & 30 & $4(3 \sim 7)$ & \\
\hline ECOG performance status & & & 0.996 \\
\hline$\leq 2$ & 13 & $5(5 \sim 8)$ & \\
\hline 3 & 24 & $5(3 \sim 10)$ & \\
\hline 4 & 26 & $4(3 \sim 9)$ & \\
\hline Antibiotic use & & & 0.640 \\
\hline No & 35 & $4(3 \sim 10)$ & \\
\hline Yes & 32 & 7 (3 9) & \\
\hline White blood cell $\left(10^{3} / \mathrm{mm}^{3}\right)$ & & & 0.655 \\
\hline$<10$ & 22 & $4(3 \sim 11)$ & \\
\hline$\geq 10$ & 45 & $5(3 \sim 8)$ & \\
\hline Hemoglobin (g/dL) & & & 0.129 \\
\hline$\geq 13$ & 15 & $7(4 \sim 15)$ & \\
\hline$<13$ & 52 & $5(3 \sim 8)$ & \\
\hline Platelet $\left(10^{3} / \mathrm{mm}^{3}\right)$ & & & 0.480 \\
\hline$\geq 150$ & 47 & $5(3 \sim 9)$ & \\
\hline$<150$ & 20 & $4(3 \sim 10)$ & \\
\hline Serum creatinine level (mg/dL) & & & 0.040 \\
\hline$\leq 1.2$ & 47 & $6(3 \sim 10)$ & \\
\hline$>1.2$ & 20 & $3(3 \sim 8)$ & \\
\hline Albumin (g/dL) & & & 0.214 \\
\hline$\geq 3.5$ & 12 & $4(2 \sim 13)$ & \\
\hline$<3.5$ & 55 & $5(3 \sim 9)$ & \\
\hline Total bilirubin (mg/dL) & & & 0.188 \\
\hline$<1.2$ & 48 & $5(3 \sim 10)$ & \\
\hline$\geq 1.2$ & 19 & $4(3 \sim 9)$ & \\
\hline Prolonged PT INR & & & 0.516 \\
\hline$\leq 1.2$ & 35 & $5(3 \sim 10)$ & \\
\hline$>1.2$ & 31 & $6(3 \sim 9)$ & \\
\hline C-reactive protein level $^{\dagger}(\mathrm{mg} / \mathrm{dL})$ & & & 0.347 \\
\hline$\leq 14.5$ & 33 & $5(3 \sim 11)$ & \\
\hline$>14.5$ & 34 & $6(3 \sim 9)$ & \\
\hline D-dimer ${ }^{\dagger}(\mu \mathrm{g} / \mathrm{mL})$ & & & 0.012 \\
\hline$<1.5$ & 33 & $7(4 \sim 13)$ & \\
\hline$\geq 1.5$ & 34 & $4(3 \sim 7)$ & \\
\hline
\end{tabular}

ECOG: Eastern Cooperative Oncology Group, PT INR: prothrombin time international normalized ratio.

*From the log-rank test, ${ }^{\dagger}$ Using the median value. 


\section{RESULTS}

\section{Patient characteristics}

The characteristics of the 67 patients and the differences between those with low $\mathrm{D}$-dimer levels $(<1.5 \mu \mathrm{g} / \mathrm{mL})$ and those with high $\mathrm{D}$-dimer levels $(\geq 1.5 \mu \mathrm{g} / \mathrm{mL})$ are shown in Table 1 . The mean age of the total study population was 71.8 years, and 30 of the patients were women (44.8\%). The most common type of cancer was lung cancer (32.8\%), followed by hepatobiliary cancer and urogenital cancer. The ECOG performance status was either 3 or 4 in approximately $80 \%$ of the patients. Antibiotics were used in $47.8 \%$ of the patients to treat a bacterial infection. The median survival time was 5 days (interquartile range, $3 \sim 10$ days). The $2 \mathrm{D}$-dimer groups showed no significant differences in characteristics except for the cancer site.

\section{Survival time by patient characteristics}

Table 2 presents differences in survival time according to the patients' characteristics. There were no statistically significant relationships between survival time and age, sex, ECOG performance status, infection, leukopenia, anemia, thrombocytopenia, hypoalbuminemia, hyperbilirubinemia, prothrombin time prolongation, and $\mathrm{C}$-reactive protein levels. Only serum creatinine and D-dimer levels showed statistically significant relationships with survival time, which was significantly shorter in patients with an elevated serum creatinine level than in those with a normal serum creatinine level (3 days vs. 6 days; $\mathrm{P}=0.040$ ) and in patients with high $\mathrm{D}$-dimer levels than in those with low $\mathrm{D}$-dimer levels (4 days vs. 7 days; $\mathrm{P}=0.012$ ). The presence of a significant difference in survival time according to D-dimer levels was also confirmed by the KaplanMeier survival analysis (Figure 1). In the univariate Cox proportional-hazards regression analysis, only $\mathrm{D}$-dimer levels were associated with a poor prognosis. Patients with high D-dimer levels were 1.83 times more likely to have a poor prognosis than those with low D-dimer levels (95\% confidence interval, 1.09 3.07; data not shown), and the difference between the 2 groups was statistically significant. Other variables, including serum creatinine levels, were not related to survival time.

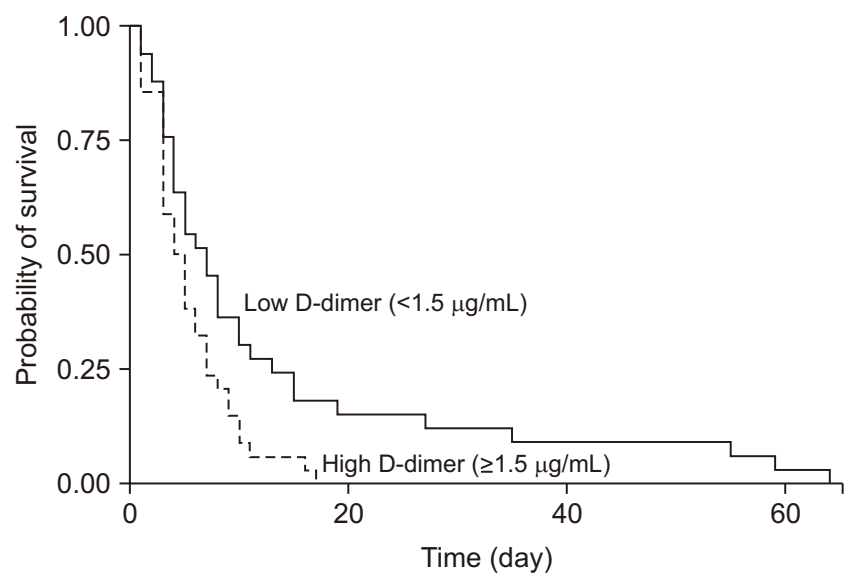

Figure 1. Kaplan - Meier survival curve according to D-dimer levels.

\section{DISCUSSION}

In this study, we confirmed the presence of a statistically significant association between $\mathrm{D}$-dimer levels and survival time in patients with terminal cancer facing imminent death. Although such an association has also been suggested in previous studies, the patients in those studies either had a single specific type of cancer or did not have terminal cancer with impending death $(3-7,12)$. Thus, the significance of this study is that it demonstrated that high $\mathrm{D}$-dimer levels were predictive of a poor prognosis in patients with terminal cancer, regardless of the type of cancer.

In this study, previously established prognostic factors, including ECOG performance status, hypoalbuminemia, hyperbilirubinemia, and a high inflammatory response, did not show a significant relationship with survival. Only elevated serum creatinine and D-dimer levels were significantly associated with survival time. This may have been because the average survival time (5 days) in our patients was short. Commonly used survival prediction systems, including the Palliative Prognostic Index, the Palliative Prognostic Score, and the Objective Prognostic Score developed in 2007 (13) are all designed to predict 3- to 4-week survival. Thus, the survival time in this study (5 days) may have been too short to evaluate the validity of the established predictors. Despite the short survival time, however, we were able to confirm the presence of an association between $\mathrm{D}$-dimer levels and survival time, indicating that it might be possible to use $\mathrm{D}$-dimer levels as a predictor of survival time in terminal cancer patients whose expected life expectancy is less than 1 week. 
Cancer is a risk factor for thrombosis. The risk of thrombosis is increased by 4.1 times (95\% confidence interval, 1.9 8.5) by cancer itself and by 6.5 times ( $95 \%$ confidence interval, 2.1 20.2) after chemotherapy $(14,15)$. However, because of the small number of patients analyzed in this study, we could not assess the risk of thrombosis according to the type of treatment. It will be necessary to evaluate whether potential differences in the risk of thrombosis according to the treatment method used affect the generalizability of our results to the broader population. Infections (e.g., pneumonia and urinary tract infections) are also known to be associated with an increased risk of thrombosis (16). In this study, we considered patients to have an infection if they were administered antibiotics, and the use of antibiotics was not related to survival time.

This study has some limitations. The first is the possibility of selection bias inherent to a retrospective analysis. The second is the limited generalizability of our results, since the data were obtained from one institution. Since this study is based on a retrospective analysis of medical records, the number of patients was relatively small (67 patients). In the palliative care unit, pain relief and improvement of symptoms are the focus of treatment; thus, several parameters-including D-dimer levels-are not examined in routine blood workups. In addition, even if an elevated D-dimer level leads to the confirmation of thrombosis, patients often refuse aggressive treatment such as surgery. Therefore, our findings should be further validated by multicenter studies involving a larger number of patients with different conditions to determine their applicability in various clinical scenarios. Care should also be taken in generalizing our findings to all patients with terminal cancer because the association between high $\mathrm{D}^{-}$-dimer levels and a poor prognosis could have been affected by the fact that $\mathrm{D}$-dimer testing was performed in patients with sudden respiratory distress in the palliative care unit. Nevertheless, unlike other clinical test results, D-dimer levels were significantly related to survival time, and the prognosis was worse in patients with high $\mathrm{D}^{-}$ dimer levels than in those with low levels. Therefore, physicians should be aware that elevated D-dimer levels in a terminal cancer patient facing imminent death may imply a poor prognosis. Third, a D-dimer level elevation may result from other causes, including myocardial infarction, sepsis, and other systemic diseases (17). However, it was difficult to distinguish the effects of other conditions on D-dimer levels from that of terminal-stage cancer because of the retrospective study design.

In conclusion, we confirmed the possibility of using D-dimer levels as a prognostic factor in terminal cancer patients facing imminent death. Further multicenter, prospective, observational studies are warranted to determine the validity of $\mathrm{D}^{-}$ dimer levels as a predictor of survival.

\section{SUPPLEMENTARY MATERIALS}

Supplementary materials can be found via https://doi. org/10.14475/kjhpc.2020.23.1.11.

\section{REFERENCES}

1. Adam SS, Key NS, Greenberg CS. D-dimer antigen: current concepts and future prospects. Blood 2009;113:2878-87.

2. Verhovsek M, Douketis JD, Yi Q, Shrivastava S, Tait RC, Baglin T, et al. Systematic review: D-dimer to predict recurrent disease after stopping anticoagulant therapy for unprovoked venous thromboembolism. Ann Intern Med 2008;149:481-90, W94.

3. Yamamoto M, Yoshinaga K, Matsuyama A, Iwasa T, Osoegawa A, Tsujita E, et al. Plasma D-dimer level as a mortality predictor in patients with advanced or recurrent colorectal cancer. Oncology 2012;83:10-5.

4. Motavaf E, Sunesen KG, Stender MT, Thorlacius-Ussing O. Prognostic value of preoperative D-dimer and carcinoembryonic antigen levels in patients undergoing intended curative resection for colorectal cancer: a prospective cohort study. Int J Colorectal Dis 2014;29:1427-32.

5. Zhou YX, Yang ZM, Feng J, Shan YJ, Wang WL, Mei YQ. High plasma D-dimer level is associated with decreased survival in patients with lung cancer: a meta-analysis. Tumour Biol 2013;34:3701-4.

6. Khoury JD, Adcock DM, Chan F, Symanowski JT, Tiefenbacher S, Goodman O, et al. Increases in quantitative D-dimer levels correlate with progressive disease better than circulating tumor cell counts in patients with refractory prostate cancer. Am J Clin Pathol 2010;134:964-9.

7. Go SI, Lee MJ, Lee WS, Choi HJ, Lee US, Kim RB, et al. D-Dimer Can Serve as a Prognostic and Predictive Biomarker for Metastatic Gastric Cancer Treated by Chemotherapy. Medicine (Baltimore) 2015;94:e951. 
8. Falanga A, Panova-Noeva M, Russo L. Procoagulant mechanisms in tumour cells. Best Pract Res Clin Haematol 2009;22:49-60.

9. Lee IC, Kim CK, Suh SY, Kim YS, Cho KH, Kang HC, et al. Validation of Scoring System for Survival Prediction in Terminal Cancer Patients in Korea. J Korean Acad Fam Med 2007;28:682-9.

10. Suh SY. Survival prediction of terminally ill cancer patients. Korean J Hosp Palliat Care 2007;10:171-7.

11. Repetto L, Fratino L, Audisio RA, Venturino A, Gianni W, Vercelli M, et al. Comprehensive geriatric assessment adds information to Eastern Cooperative Oncology Group performance status in elderly cancer patients: an Italian Group for Geriatric Oncology Study. J Clin Oncol 2002;20:494-502.

12. den Daas N. Estimating length of survival in end-stage cancer: a review of the literature. J Pain Symptom Manage 1995;10:548-55.

13. Park HJ, Choi YS, Kim SM, Kim JE, Kim EH, Kim EY, et al. Validation of the objective prognostic score for terminal cancer patients in a tertiary hospital. Korean J Clin Geri 2016;17:59-67.

14. Rao BB, Kalayarasan R, Kate V, Ananthakrishnan N. Venous thromboembolism in cancer patients undergoing major abdominal surgery: Prevention and management. Int Sch Res Notices 2012;2012:1-22.

15. Heit JA, Silverstein MD, Mohr DN, Petterson TM, O'Fallon WM, Melton L, 3rd. Risk factors for deep vein thrombosis and pulmonary embolism: a population-based case-control study. Arch Intern Med 2000;160:809-15.

16. Tichelaar YI, Kluin-Nelemans HJ, Meijer K. Infections and inflammatory diseases as risk factors for venous thrombosis. A systematic review. Thromb Haemost 2012;107:827-37.

17. Bounameaux H, de Moerloose P, Perrier A, Reber G. Plasma measurement of D-dimer as diagnostic aid in suspected venous thromboembolism: an overview. Thromb Haemost 1994;71:1-6. 